\title{
A Local Limit Theorem in the Theory of Overpartitions ${ }^{1}$
}

\author{
Sylvie Corteel, ${ }^{2}$ William M. Y. Goh, ${ }^{3}$ and Pawel Hitczenko ${ }^{3}$
}

\begin{abstract}
An overpartition of an integer $n$ is a partition where the last occurrence of a part can be overlined. We study the weight of the overlined parts of an overpartition counted with or without their multiplicities. This is a continuation of a work by Corteel and Hitczenko where it was shown that the expected weight of the overlined parts is asymptotic to $n / 3$ as $n \rightarrow \infty$ and that the expected weight of the overlined parts counted with multiplicity is $n / 2$. Here we refine these results. We first compute the asymptotics of the variance of the weight of the overlined parts counted with multiplicity. We then asymptotically evaluate the probability that the weight of the overlined parts is $n / 3 \pm k$ for $k=o(n)$ and the probability that the weight of the overlined parts counted with multiplicity is $n / 2 \pm k$ for $k=o(n)$. The first computation is straightforward and uses known asymptotics of partitions. The second one is more involved and requires a sieve argument and the application of the saddle-point method. From that we can directly evaluate the probability that two random partitions of $n$ do not share a part.
\end{abstract}

Key Words. Partitions, Combinatorial probability, Local limit theorem, Asymptotic analysis.

1. Introduction. An overpartition of the integer $n$ is a non-increasing sequence of positive integers that sum to $n$ where the last occurrence of an integer can be overlined. The quantity $n$ is called the weight. Given an overpartition $\left(\lambda_{1}, \ldots, \lambda_{k}\right)$ we call the $\lambda_{i}$ 's the parts. The number of part sizes is the number of distinct integers that occur in the overpartition. The multiplicity of part $i$ is the number of occurrences of part $i$ in $\lambda$ (overlined or not). The weight of the overlined parts is the sum of the overlined parts and the weight of the overlined parts counted with multiplicity is the sum of the sizes of the overlined parts multiplied by their multiplicity. For example, $\lambda=(5, \overline{4}, 2,2, \overline{2}, 1)$ is an overpartition of 16. It has six parts, the multiplicity of part 2 is 3 . The weight of the overlined parts is 6 and the weight of the overlined parts counted with their multiplicities is $10=4+2 \cdot 3$. We denote by $\bar{p}(n)$ the number of overpartitions of $n$.

Overpartitions were named by Corteel and Lovejoy [10], [12], [13] and used to give the first combinatorial proofs of the $q$-Gauss identity and Ramanujan's ${ }_{1} \psi_{1}$ summation [12]. They have been studied using combinatorial, $q$-series, and number theoretical techniques under different names and guises (superpartitions, jagged partitions, joint partitions, 2modular diagrams,... ) [4], [6], [14], [18], [22], [23], [24], [27]. First asymptotic and probabilistic results on overpartitions were presented in [11].

\footnotetext{
${ }^{1}$ This work was supported in part by NSF Grant INT-0230800. The work of the third author was also partially supported by NSA Grants MSPF-02G-043 and MSPF-04G-054.

${ }^{2}$ CNRS LRI, Université Paris-Sud, Orsay, France. corteel@1ri.fr.

${ }^{3}$ Department of Mathematics, Drexel University, Philadelphia, PA 19104, USA. \{wgoh,phitczenko\}@ mcs.drexel.edu.
}

Received January 14, 2005. Communicated by P. Jacquet, D. Panario, and W. Szpankowski.

Online publication December 20, 2006. 
The purpose of this work is to study the weight of the overlined parts counted with, or without, their multiplicity. As is customary we consider the uniform probability measure on the set of all overpartitions of $n$, and by random overpartition of $n$ we mean an overpartition picked according to that measure. We denote by $\bar{W}_{n}$ (resp. $\bar{M}_{n}$ ) the weight of the overlined parts (resp. counted with their multiplicity) of a random overpartition of $n$.

From Theorem 1.4 of [11] we have information on the expectation of these variables.

LEMMA 1. The expected weight $\mathrm{E} \bar{W}_{n}$ of the overlined parts of an overpartition of $n$ is asymptotically equivalent to $n / 3$ as $n$ goes to infinity.

LEMMA 2. The expected weight $\mathrm{E} \bar{M}_{n}$ of the overlined parts counted with their multiplicity of an overpartition of $n$ is $n / 2$.

The first lemma is only asymptotic. The second lemma is true for any $n$, thanks to an easy combinatorial involution presented in Section 2 below. Of course one may hope for more precise information about these two random variables than merely their expected values. Surprisingly it is very easy to obtain more information on $\bar{W}_{n}$ and we present these results in Section 2. On the contrary it seems much harder to find out more about $\bar{M}_{n}$ and this is the main task of the paper. We first present some probabilistic arguments to study the variance of $\bar{M}_{n}$ :

THEOREM 1. The variance of the weight of the overlined parts, counted with multiplicities, in a random overpartition of $n$ satisfies

$$
\operatorname{var}\left(\bar{M}_{n}\right) \sim \frac{4}{3 \pi} n^{3 / 2},
$$

as $n \rightarrow \infty$.

Our ultimate goal, however, is to establish a central limit theorem for $\bar{M}_{n}$. In fact, we will obtain a strong version, usually referred to as a local limit theorem in probabilistic literature.

By means of heuristic introduction, as to what one might expect let us suppose that $\left(Y_{n}\right)$ is a sequence of integer-valued random variables such that $\mathrm{E} Y_{n}=0$ and $\sigma_{n}^{2}=\operatorname{var}\left(Y_{n}\right)<\infty$. If the normalized sequence $Y_{n} / \sigma_{n}$ satisfies a (strong version of the) central limit theorem then, generally speaking, we expect that

$$
\mathrm{P}\left(Y_{n}=k\right) \sim \frac{e^{-k^{2} / 2 \sigma_{n}^{2}}}{\sqrt{2 \pi} \sigma_{n}},
$$

for $k$ satisfying $-k_{n} \leq k \leq k_{n}$. Of particular interest is a situation where $k_{n}$ may be chosen so that $\operatorname{var}\left(Y_{n}\right) / k_{n}^{2} \rightarrow 0$ as $n \rightarrow \infty$, since then, by Chebyshev's inequality, the probabilities (1) cover essentially the whole mass of the distribution of $Y_{n}$. (In our case since the variance of $\bar{M}_{n}$ is of order $n^{3 / 2}$ we would like $k_{n}$ to go to infinity at a rate faster than $n^{3 / 4}$.) Perhaps the oldest and best known result of this type is the celebrated De Moivre-Laplace theorem. We refer the reader to Chapter VII, Sections 2 and 3, of 
[17] or Chapter I, Section 6, of [25] for nice presentations of that theorem and to [8] for an example of a general local limit theorem.

We now turn to our specific situation and consider $\bar{M}_{2 n}$. (Using only even indices is purely for convenience; it assures that the mean value of $\bar{M}_{2 n}$ is an integer and thus is taken on by $\bar{M}_{2 n}$.) We apply our heuristics to $Y_{n}=\bar{M}_{2 n}-n$. Since by Theorem 1 $\operatorname{var}\left(\bar{M}_{2 n}\right) \sim(8 \sqrt{2} / 3 \pi) n^{3 / 2}$, we expect $\mathrm{P}\left(\bar{M}_{2 n}=n+k\right)$ to satisfy

$$
\mathrm{P}\left(\bar{M}_{2 n}=n+k\right) \sim \frac{e^{-k^{2} / 2 \sigma_{2 n}^{2}}}{\sqrt{2 \pi} \sigma_{2 n}} \sim \frac{\sqrt{3} e^{-3 \pi k^{2} / 16 \sqrt{2} n^{3 / 2}}}{4 \sqrt[4]{2} n^{3 / 4}}
$$

in a certain range of $k$ 's. This is exactly what the next theorem asserts.

THEOREM 2. As long as $k=o(n)$, uniformly over $k$ we have

$$
\mathrm{P}\left(\bar{M}_{2 n}=n+k\right)=\frac{\sqrt{3} e^{-3 \pi k^{2} / 16 \sqrt{2} n^{3 / 2}}}{4 \sqrt[4]{2} n^{3 / 4}}(1+o(1)) .
$$

We propose a combinatorial setting of the problem and some combinatorial applications in Section 2. For example we find the asymptotic value of the probability that two random partitions of $n$ do not share a part as $n \rightarrow \infty$. Then we prove Theorems 1 and 2 in Sections 3 and 4 using asymptotic and probabilistic techniques.

Our approach is based on asymptotic methods that are ubiquitous in the analysis of algorithms and combinatorial structures [2], [19], [20], [26]. From the point of view of methods, technically the most difficult part of this work is presented in Section 4 where we asymptotically evaluate an alternating sum given in Proposition 1 below (a particular case of that sum appeared in [15] in the context of a pentagonal number sieve). Our approach is based on methods detailed in [16]; we represent our sum as a double contour integral. Many other examples of such representations are given in [16] (see Chapter 5). However, the main focus of [16] is on how such representations can be used to obtain interesting combinatorial identities, and provides no information about asymptotic analysis of these expressions. Thus, it is not clear how such representations can be used to obtain useful asymptotic results. Our method of handling a double contour integral offers a practical solution that, we hope, will find applications elsewhere. The idea is straightforward: we use the inner integral to obtain a "uniform"approximation in the region that gives the major contribution to the outer integral. In a sense, we work out asymptotics twice in a row; once for the inner integral and then for the outer one. The desired asymptotic result follows by applying "traditional" asymptotic methods (in our specific case we rely on the saddle-point method).

2. Combinatorial Setting and Applications. From the definition of overpartitions, the following facts are obvious:

- There is a bijection between overpartitions of $n$ whose weight of the overlined parts is $\ell$ and pairs of partitions of $\ell$ and $n-\ell$ where the first one is into distinct parts.

- There is a bijection between overpartitions of $n$ whose weight of the overlined parts counted with multiplicity is $\ell$ and pairs of partitions of $\ell$ and $n-\ell$ that do not share a part. 
The first bijection is as follows. Given an overpartition, we put all the overlined parts in one partition and the rest in the other partition. For example, starting with the overpartition $(8,6,6,4,4, \overline{4}, 2, \overline{1})$, we get $(4,1)$ and $(8,6,6,4,4,2)$.

The second bijection is as simple. Given an overpartition, we put all the copies of the overlined part sizes in one partition and the rest in the other partition. For example, starting with the overpartition $(8,6,6,4,4, \overline{4}, 2, \overline{1})$, we get $(4,4,4,1)$ and $(8,6,6,2)$.

We now interpret these two facts in a probabilistic context and exploit their consequences.

2.1. Overpartitions and Pairs of Partitions where the First One Is into Distinct Parts. Let $d(n)$ (resp. $p(n)$ ) be the number of partitions into distinct parts (resp. ordinary partitions) of $n$. The first fact tells us that the number of overpartitions of $n$ where the weight of the overlined parts is $\ell$ is equal to $d(\ell) p(n-\ell)$. Since, clearly,

$$
\bar{p}(n)=\sum_{\ell=0}^{n} d(\ell) p(n-\ell),
$$

we can write

$$
\mathrm{P}\left(\bar{W}_{n}=\ell\right)=\frac{d(\ell) p(n-\ell)}{\bar{p}(n)} .
$$

The asymptotic behaviors of $d(n), p(n)$, and $\bar{p}(n)$ are well known, see Chapter 6 of [2] for example. Since, by Lemma 1 , the expected value of $\bar{W}_{n}$ is about $n / 3$, it makes sense to consider $\ell$ of the form $\lfloor n / 3\rfloor \pm k$. We can easily get the following local limit theorem:

THEOREM 3. For $k=o(n)$,

$$
\mathrm{P}\left(\bar{W}_{n}=\lfloor n / 3\rfloor \pm k\right)=\frac{3}{4 n^{3 / 4}} \exp \left(\frac{-9 \pi k^{2}}{16 n^{3 / 2}}\right)(1+o(1))
$$

as $n \rightarrow \infty$.

PROOF. According to a general theorem of Meinardus [2, Chapter 6] we have

$$
\begin{aligned}
& d(n)=\frac{1}{4 n^{3 / 4} 3^{1 / 4}} e^{\pi \sqrt{n / 3}}\left(1+O\left(\frac{1}{n^{1 / 2}-\delta}\right)\right), \\
& p(n)=\frac{1}{4 \sqrt{3} n} e^{\pi \sqrt{2 n / 3}}\left(1+O\left(\frac{1}{n^{1 / 2}}\right)\right), \\
& \bar{p}(n)=\frac{1}{8 n} e^{\pi \sqrt{n}}\left(1+O\left(\frac{1}{n^{1 / 2}}\right)\right) .
\end{aligned}
$$

When $k=o(n)$,

$$
\frac{8 n}{4(n / 3+k)^{3 / 4} 3^{1 / 4} 4 \sqrt{3}(2 n / 3-k)}=\frac{3}{4 n^{3 / 4}}\left(1+O\left(\frac{k}{n}\right)\right)
$$


and

$$
\sqrt{(n / 3 \pm k) / 3}+\sqrt{2(2 n / 3 \pm k) / 3}-\sqrt{n}=-\frac{9 k^{2}}{16 n^{3 / 2}}-\frac{27 k^{3}}{64 n^{5 / 2}}+O\left(\frac{k^{4}}{n^{7 / 2}}\right) .
$$

Then we get

$$
\frac{d(\lfloor n / 3\rfloor+k) p(\lfloor 2 n / 3\rfloor-k)}{\bar{p}(n)}=\frac{3}{4 n^{3 / 4}} \exp \left(\frac{-9 k^{2}}{16 n^{3 / 2}}\right)(1+o(1)) .
$$

This result implies that

COROLLARY 1.

$$
\operatorname{var}\left(\bar{W}_{n}\right) \sim \frac{8 n^{3 / 2}}{9 \pi} .
$$

2.2. Overpartitions and Pairs of Partitions that Do Not Share a Part. The second bijection described at the beginning of this section implies that the expected weight of the overlined parts counted with their multiplicity of an overpartition of $n$ is $n / 2$ as we can apply the bijection, switch the two partitions, and apply the reverse bijection. This is equivalent to the following involution on overpartitions. Given an overpartition, list all its part sizes. Overline the ones that are non-overlined and remove the overline from the overlined parts. For example, starting with the overpartition $(8,6,6,4,4, \overline{4}, 2, \overline{1})$, we get $(\overline{8}, 6, \overline{6}, 4,4,4, \overline{2}, 1)$.

This second fact also implies a recursive formula for the number $\bar{p}(n, \ell)$ of overpartitions of $n$ whose weight of the overlined parts counted with multiplicity is $\ell$. The case $n=2 k$ and $\ell=k$ was treated in [15]. We apply the same sieve argument as in [15] and get

PROPOSITION 1.

$$
\bar{p}(n, \ell)=\sum_{j=-\infty}^{\infty}(-1)^{j} p\left(\ell-\frac{3 j^{2}+j}{2}\right) p\left(n-\ell-\frac{3 j^{2}+j}{2}\right) .
$$

In terms of generating functions we can write

$$
\sum_{n \geq \ell} \bar{p}(n, \ell) q^{n}=\prod_{i \geq 1}\left(1-q^{i}\right) \sum_{n \geq \ell} p(\ell) p(n-\ell) q^{n} .
$$

This corresponds to equation (2) in [15] which was a starting point for a proof of an analog of Proposition 1.

To set a stage for the proof of Theorem 2, we follow the same reasoning as in the previous subsection, except that we use the second bijection. First, it is clear that $\bar{p}(n, \ell)=\bar{p}(n, n-\ell)$, and that

$$
\bar{p}(2 n)=\sum_{\ell=0}^{2 n} \bar{p}(2 n, \ell)=\bar{p}(2 n, n)+2 \sum_{\ell=0}^{n-1} \bar{p}(2 n, \ell) .
$$


Hence

$$
\mathrm{P}\left(\bar{M}_{2 n}=\ell\right)=\frac{\bar{p}(2 n, \ell)}{\bar{p}(2 n)} .
$$

Since the asymptotic behavior of $\bar{p}(2 n)$ is well known (see (5)), we focus on the numerator. Furthermore, since $\mathrm{E} \bar{M}_{2 n}=n$ we consider $\ell=n+k$ and in Section 4 below, we compute the asymptotic formula for $\bar{p}(2 n, n+k)$ with $k=o(n)$. This formula is

$$
\bar{p}(2 n, n+k) \sim \frac{\sqrt{3}}{2^{25 / 4} n^{7 / 4}} e^{\pi \sqrt{2 n}} e^{-3 \pi k^{2} / 16 \sqrt{2} n^{3 / 2}},
$$

as $n \rightarrow \infty$.

In particular, $\bar{p}(2 n, n)$ is the number of pairs of partitions of $n$ that do not share a part denoted by $p_{2}(n)$ in [15]. Thus,

$$
\frac{\bar{p}(2 n, n)}{p^{2}(n)}
$$

is the probability that two randomly and independently chosen partitions of $n$ do not share a part. We then just need to apply the asymptotic formula (4) for $p(n)$ and we get

COROLLARY 2. The probability that two independently chosen random partitions of $n$ do not share a part is asymptotically equivalent to

$$
\frac{3 \sqrt{3} n^{1 / 4}}{4 \cdot 2^{1 / 4}} e^{\pi \sqrt{2 n}(1-2 / \sqrt{3})} .
$$

3. Proof of Theorem 1. Let $m_{j}$ be a random variable that counts the multiplicity of part size $j$ in a random overpartition of $n$. Thus, the constraint on the values of $m_{j}$ is that

$$
\sum_{j \geq 1} j m_{j}=n .
$$

It follows directly from the definition of overpartitions that the weight of overlined parts counted with multiplicity can be probabilistically represented as

$$
\bar{M}_{n} \stackrel{d}{=} \sum_{j \geq 1} j m_{j} \varepsilon_{j}
$$

where " $=$ " denotes the equality in distribution, $\left(\varepsilon_{j}\right)$ is a sequence of independent identically distributed random variables with values in $\{0,1\}$ satisfying $P\left(\varepsilon_{j}=0\right)=\frac{1}{2}$, and furthermore the sequences $\left(m_{j}\right)$ and $\left(\varepsilon_{j}\right)$ are independent (basically, $\varepsilon_{j}$ indicates whether the last occurrence of $j$ is overlined or not). To find the asymptotics of the variance of $\bar{M}_{n}$ we use the following simple fact (its proof is short, elementary, and omitted; it uses only basic properties of the conditional expectations [7, Theorem 4.4.2]).

LEMMA 3. For any random variable $X$ and a $\sigma$-algebra $\mathcal{F}$ we have

$$
\operatorname{var}(X)=\operatorname{Evar}_{\mathcal{F}}(X)+\operatorname{var}(\mathrm{E}(X \mid \mathcal{F})),
$$

where $\operatorname{var}_{\mathcal{F}}(X)=\mathrm{E}\left((X-\mathrm{E}(X \mid \mathcal{F}))^{2} \mid \mathcal{F}\right)$ and $\mathrm{E}(X \mid \mathcal{F})$ is the conditional expectation of $X$ given $\mathcal{F}$. 
Applying this to $\bar{M}_{n}$ and $\mathcal{F}=\sigma\left\{m_{j}: j \geq 1\right\}$ we see that

$$
\mathrm{E}\left(\bar{M}_{n} \mid \mathcal{F}\right)=\frac{1}{2} \sum_{j \geq 1} j m_{j}=\frac{n}{2}
$$

so that

$$
\operatorname{var}\left(\mathrm{E}\left(\bar{M}_{n} \mid \mathcal{F}\right)\right)=0 .
$$

Further, by the $\mathcal{F}$-measurability of the $m_{j}$ 's and their independence of $\left(\varepsilon_{j}\right)$,

$$
\operatorname{var}_{\mathcal{F}}\left(\bar{M}_{n}\right)=\sum_{j \geq 1} j^{2} m_{j}^{2} \operatorname{var}\left(\varepsilon_{j}\right)=\frac{1}{4} \sum_{j \geq 1} j^{2} m_{j}^{2}
$$

Hence,

$$
\operatorname{var}\left(\bar{M}_{n}\right)=\frac{1}{4} \sum_{j \geq 1} j^{2} \mathrm{E} m_{j}^{2}
$$

LEMMA 4. Let $X$ be a random variable whose values are in the set of positive integers. Then

$$
\mathrm{E} X^{2}=\sum_{m \geq 1}(2 m-1) \mathrm{P}(X \geq m)
$$

Proof. We have

$$
\mathrm{E} X^{2}=\sum_{m \geq 1} m^{2} \mathrm{P}(X=m)=\sum_{m \geq 1} m^{2}(\mathrm{P}(X \geq m)-\mathrm{P}(X \geq m+1)) .
$$

Changing the order of summation and shifting the index by one gives the statement.

In order to proceed we recall that according to Lemma 2.2 of [11]

$$
\mathrm{P}\left(m_{j} \geq m\right)=\frac{2}{\bar{p}(n)} \sum_{i \geq 0}(-1)^{i} \bar{p}(n-(m+i) j) .
$$

Since (5) yields, for example,

$$
\frac{\bar{p}(n-k i)}{\bar{p}(n)}= \begin{cases}\left(1+O\left(1 / n^{1 / 4}\right)\right) e^{-\pi k i / 2 \sqrt{n}}, & \text { if } k i \leq n^{3 / 4} \\ O\left(e^{-\pi n^{1 / 4} / 2}\right), & \text { otherwise }\end{cases}
$$

applying Lemma 4 to $X=m_{j}$ and using (7) and (8) we get

$$
\begin{aligned}
\mathrm{E} m_{j}^{2} & =\sum_{m \geq 1}(2 m-1) \mathrm{P}\left(m_{j} \geq m\right) \\
& =\sum_{m \geq 1}(2 m-1) \cdot 2 \sum_{i \geq 0}(-1)^{i} \frac{\bar{p}(n-(m+i) j)}{\bar{p}(n)}
\end{aligned}
$$




$$
\begin{aligned}
& \sim 2 \sum_{m \geq 1}(2 m-1) \sum_{i \geq 0}(-1)^{i} \exp \left(-\frac{\pi}{2 \sqrt{n}}(m j+i j)\right) \\
& =2 \sum_{m \geq 1}(2 m-1) \exp \left(-\frac{\pi m j}{2 \sqrt{n}}\right) \sum_{i \geq 0}(-1)^{i} \exp \left(-\frac{\pi}{2 \sqrt{n}} i j\right) \\
& =2 \frac{\exp (-\pi j / 2 \sqrt{n})(1+\exp (-\pi j / 2 \sqrt{n}))}{(1-\exp (-\pi j / 2 \sqrt{n}))^{2}} \cdot \frac{1}{1+\exp (-\pi j / 2 \sqrt{n})} \\
& =2 \frac{\exp (-\pi j / 2 \sqrt{n})}{(1-\exp (-\pi j / 2 \sqrt{n}))^{2}},
\end{aligned}
$$

where in the penultimate step we used the fact that for any $0<q<1$,

$$
\sum_{m \geq 1}(2 m-1) q^{m}=\frac{q(1+q)}{(1-q)^{2}},
$$

and step (9) will be justified momentarily. So,

$$
\text { (11) } \begin{aligned}
\operatorname{var}\left(\bar{M}_{n}\right) & =\frac{1}{4} \sum_{j \geq 1} j^{2} \mathrm{E} m_{j}^{2}=\frac{1}{2} \sum_{j \geq 1} j^{2} \frac{\exp (-\pi j / 2 \sqrt{n})}{(1-\exp (-\pi j / 2 \sqrt{n}))^{2}} \\
& =\frac{1}{2} \int_{0}^{\infty} x^{2} \frac{\exp (-\pi x / 2 \sqrt{n})}{(1-\exp (-\pi x / 2 \sqrt{n}))^{2}} d x+O(n) \\
& \sim \frac{1}{2}\left(\frac{2 n^{1 / 2}}{\pi}\right)^{3} \int_{0}^{\infty} \frac{y^{2} \exp (-y)}{(1-\exp (-y))^{2}} d y=\frac{4 n^{3 / 2}}{\pi^{3}} \cdot \frac{\pi^{2}}{3}=\frac{4 n^{3 / 2}}{3 \pi} .
\end{aligned}
$$

Step (9) is valid since the error incurred by applying (8) results in $(-1)^{i}$ in (9) being replaced by

$$
(-1)^{i}+O\left(\frac{1}{n^{1 / 4}}\right)
$$

which in turn results in (10) being replaced by

$$
2 \frac{\exp (-\pi j / 2 \sqrt{n})}{(1-\exp (-\pi j / 2 \sqrt{n}))^{2}}+O\left(\frac{1}{n^{1 / 4}}\right) \cdot \frac{\exp (-\pi j / 2 \sqrt{n})(1+\exp (-\pi j / 2 \sqrt{n}))}{(1-\exp (-\pi j / 2 \sqrt{n}))^{3}} .
$$

Consequently, the error in (11) is of order

$$
n^{3 / 2-1 / 4} \int_{\pi /(2 \sqrt{n})}^{\infty} \frac{y^{2} e^{-y}\left(1+e^{-y}\right)}{\left(1-\mathrm{e}^{-y}\right)^{3}} d y=O\left(n^{5 / 4} \log n\right) .
$$

We refer the reader to [11] for a more detailed presentation of similar calculations.

4. Proof of Theorem 2. As we explained earlier, in order to complete the proof of Theorem 2 it is enough to justify (6). In view of Proposition 1, this amounts to evaluating 
asymptotically the sum

$$
\sum_{j=-\infty}^{\infty}(-1)^{j} p\left(n-k-\frac{3 j^{2}+j}{2}\right) p\left(n+k-\frac{3 j^{2}+j}{2}\right) .
$$

We begin by finding a generating function for $p(n-k) p(n+k)$ and our starting point is the well-known formula

$$
f(x):=\sum_{n \geq 0} p(n) x^{n}=\prod_{j \geq 1} \frac{1}{\left(1-x^{j}\right)}, \quad \text { for } \quad|x|<1 .
$$

For all $|\xi|<1$, we choose $x$ with $|x|>1$ such that $|x \xi|<1$ and form

$$
f(x \xi) f\left(x^{-1}\right)=\sum_{n \geq 0} \sum_{m \geq 0} p(n) p(m) \xi^{n} x^{n-m} .
$$

Multiplying this equation by $x^{2 k-1}$ gives

$$
x^{2 k-1} f(x \xi) f\left(x^{-1}\right)=\sum_{n \geq 0} \sum_{m \geq 0} p(n) p(m) \xi^{n} x^{n+2 k-m-1} .
$$

Term-by-term integration along the circle $C_{1}:|x|=r>1$ with $|r \xi|<1$ is justified and we have

$$
F_{k}(\xi):=\sum_{n \geq 0} p(n) p(n+2 k) \xi^{n}=\frac{1}{2 \pi i} \oint_{C_{1}} x^{2 k-1} f(x \xi) f\left(x^{-1}\right) d x,
$$

for $|\xi|<1$. By the Cauchy residue theorem

$$
p(n) p(n+2 k)=\frac{1}{2 \pi i} \oint_{|\xi|=\rho} \frac{F_{k}(\xi)}{\xi^{n+1}} d \xi,
$$

or

$$
p(n-k) p(n+k)=\frac{1}{2 \pi i} \oint_{|\xi|=\rho} \frac{F_{k}(\xi)}{\xi^{n-k+1}} d \xi,
$$

where $\rho$ is any number less than 1 and $n \neq 0$. The precise value for $\rho$ will become clear later. We now multiply (13) by $(-1)^{j}$, replace $n$ by $n-\left(3 j^{2}+j\right) / 2$ and sum up with respect to $j$ to get

$$
\begin{array}{rl}
\sum_{j=-\infty}^{\infty}(-1)^{j} & p\left(n-k-\frac{3 j^{2}+j}{2}\right) p\left(n+k-\frac{3 j^{2}+j}{2}\right) \\
= & \frac{1}{2 \pi i} \oint_{|\xi|=\rho} \frac{F_{k}(\xi)\left(\sum_{j=-\infty}^{\infty}(-1)^{j} \xi^{\left(3 j^{2}+j\right) / 2}\right)}{\xi^{n-k+1}} d \xi
\end{array}
$$

In deriving the above equation, because of absolute convergence, the interchange of integration and summation is justified. The convention that $p(n)$ is zero when $n$ is negative is automatically fulfilled, for in (13) any such function $p$ is zero by the Cauchy 
integral theorem. We now use an identity (see, for example, Theorem 353 of [21] or Theorem 1.2 and Exercise 26 in Chapter III of [3]): for $|x|<1$,

$$
\sum_{j=-\infty}^{\infty}(-1)^{j} x^{\left(3 j^{2}+j\right) / 2}=\prod_{j \geq 1}\left(1-x^{j}\right)=\frac{1}{f(x)} .
$$

Substituting this into the last integral we get with $\rho<1$,

$$
\begin{gathered}
\sum_{j=-\infty}^{\infty}(-1)^{j} p\left(n-k-\frac{3 j^{2}+j}{2}\right) p\left(n+k-\frac{3 j^{2}+j}{2}\right) \\
=\frac{1}{2 \pi i} \oint_{|\xi|=\rho} \frac{F_{k}(\xi)}{f(\xi) \xi^{n-k+1}} d \xi .
\end{gathered}
$$

In order to complete the argument, we need to evaluate this integral asymptotically. Note that for positive $\xi, F_{k}(\xi)$ and $f(\xi)$ are also positive so that the integrand $F_{k}(\xi) / f(\xi)$ is a positive function with a strong singularity at $\xi=1$. The major contribution to the integral (14) is expected to come from an immediate neighborhood of $\xi=1$. Our analysis is carried out in two steps. First, we use the integral representation (12) of $F_{k}(\xi)$ and a functional equation for $f(\xi)$ to determine the asymptotic behavior of the integrand near $\xi=1$. We then use the saddle-point method to analyze the integral (14). We carry out the details in two separate subsections below, beginning with $F_{k}(\xi)$.

4.1. Asymptotics of the Integrand. We are going to find the asymptotics for $F_{k}(\xi)$ as $\xi \rightarrow 1$ or equivalently for $F_{k}\left(e^{-2 \pi s}\right)$ as $s \rightarrow 0^{+}$by applying the saddle-point method to the integral in (12). Change of variables in (12) yields

$$
F_{k}(\xi)=\frac{1}{2 \pi i} \oint_{|x|=r<1,|\xi / x|<1} x^{-2 k-1} f\left(\frac{\xi}{x}\right) f(x) d x .
$$

Our next step is a parallel presentation of Ayoub [3, Chapter III, Section 2], who used a representation

$$
p(n)=\frac{1}{2 \pi i} \oint_{C} \frac{f(x)}{x^{n+1}} d x
$$

to obtain the Hardy-Ramanujan result on the asymptotics of the partition function (Ayoub credits this approach to $\mathrm{J}$. $\mathrm{V}$. Uspensky). The basic idea is to use a functional equation for $f(z)$; writing $z$ as $e^{-2 \pi w}$, with $\operatorname{Re}(w)>0$, we have (see, e.g., Theorem 3.2 in Chapter III of [3])

$$
f\left(e^{-2 \pi w}\right)=\sqrt{w} e^{(\pi / 12)(1 / w-w)} f\left(e^{-2 \pi / w}\right) .
$$

Since $f\left(e^{-2 \pi / w}\right) \rightarrow 1$ very rapidly as $w \rightarrow 0^{+}$, the behavior of $f\left(e^{-2 \pi w}\right)$ is dictated by $\sqrt{w} e^{(\pi / 12)(1 / w-w)}$. After estimating the error terms, one may replace the integrand in (15) by these new expressions. The resulting integral is much easier to evaluate and we use the saddle-point method to do this. We refer to [3] for more details on how $\sqrt{w} e^{(\pi / 12)(1 / w-w)}$ can be used in place of $f\left(e^{-2 \pi w}\right)$ and how to handle the error terms. We suppress these tedious estimations here and concentrate on the leading term. 
Write $x=e^{-2 \pi \tau}$ and $\xi=e^{-2 \pi s}$, where both $\operatorname{Re}(\tau)$ and $\operatorname{Re}(s)$ are positive. The condition $|\xi / x|<1$ on the contour in (15) translates into

$$
\operatorname{Re}(s)>\operatorname{Re}(\tau)>0 .
$$

Use the variable $\tau$ as the integration variable to get

$$
F_{k}(\xi)=F_{k}\left(e^{-2 \pi s}\right)=(-i) \int_{L} e^{4 \pi k \tau} f\left(e^{-2 \pi \tau}\right) f\left(e^{-2 \pi s} e^{2 \pi \tau}\right) d \tau,
$$

where the contour $L$ is an upward vertical line segment with $\operatorname{Re}(L)>0$ and $\operatorname{Im}(L)$ is between $\pm \frac{1}{2}$. Here we have the freedom to vary $\operatorname{Re}(L)>0$ as long as we keep it less than $\operatorname{Re}(s)$. In what follows, it will become clear that in order to incorporate the uniformity of $k$, the contour $L$ is chosen such that $\operatorname{Re}(L) \sim \operatorname{Re}(s) / 2$. We now apply the functional equation (16) to $f\left(e^{-2 \pi \tau}\right)$ and $f\left(e^{-2 \pi s} e^{2 \pi \tau}\right)$ and substitute the result into the last integral. We get

$$
\begin{aligned}
& F_{k}\left(e^{-2 \pi s}\right) \\
& \quad=(-i) \int_{L} \sqrt{\tau} \sqrt{s-\tau} e^{(\pi / 12)[(1 / \tau-\tau)+1 /(s-\tau)-(s-\tau)]} e^{4 \pi k \tau} f\left(e^{-2 \pi / \tau}\right) f\left(e^{-2 \pi /(s-\tau)}\right) d \tau .
\end{aligned}
$$

Since both $f\left(e^{-2 \pi / \tau}\right)$ and $f\left(e^{-2 \pi /(s-\tau)}\right) \rightarrow 1$ rapidly as $s \rightarrow 0^{+}$, they can be dropped from the integrand without affecting the asymptotics:

$$
\begin{aligned}
F_{k}\left(e^{-2 \pi s}\right) & \sim(-i) \int_{L} \sqrt{\tau} \sqrt{s-\tau} e^{(\pi / 12)[(1 / \tau-\tau)+1 /(s-\tau)-(s-\tau)]} e^{4 \pi k \tau} d \tau \\
& =(-i) e^{-\pi s / 12} \int_{L} \sqrt{\tau} \sqrt{s-\tau} e^{(\pi / 12)(1 / \tau+1 /(s-\tau)+48 k \tau)} d \tau .
\end{aligned}
$$

This is the integral where we start to apply the saddle-point method. Note that, because of the form of the integrand in (17), the saddle-point will depend on $s$ as well as $k$. Define

$$
h(\tau):=\frac{1}{\tau}+\frac{1}{s-\tau}+48 k \tau .
$$

Set $h^{\prime}(\tau)=0$ and solve it for $\tau$ to get the saddle point:

$$
h^{\prime}(\tau)=\frac{-1}{\tau^{2}}+\frac{1}{(s-\tau)^{2}}+48 k=0 .
$$

This implies that

$$
-(s-\tau)^{2}+\tau^{2}+48 k \tau^{2}(s-\tau)^{2}=0 .
$$

To solve it for $\tau$, we write $\tau:=s u$ and proceed to determine the equation satisfied by $u$. The above equation is reduced to

$$
2 u+48\left(k s^{2}\right) u^{2}(1-u)^{2}=1 .
$$


This equation shows that the root $u$ depends only on $k s^{2}$. So let us treat $u=u\left(k s^{2}\right)$ as a function of $k s^{2}$; the saddle point in question is just $s u$. We now assume that $k$ is a parameter satisfying

$$
k s^{2} \rightarrow 0, \quad \text { as } \quad s \rightarrow 0 .
$$

Later we will see that $s$ is $\Theta(1 / \sqrt{n})$ and the assumption that $k=o(n)$ will validify the above equation. Having said this it is useful to point out the following:

(i) As $k s^{2} \rightarrow 0$, (18) has a unique positive root. This is the solution that is used in the saddle-point method. Let this positive solution be denoted by $u$. We have further:

(ii) $\lim _{k s^{2} \rightarrow 0} u=\frac{1}{2}$.

(iii) $u=\frac{1}{2}-\frac{3}{2}\left(k s^{2}\right)+o\left(k s^{2}\right)$, as $k s^{2} \rightarrow 0$.

We comment that (ii) and (iii) can be formally justified by "bootstrapping"from (18). Namely, setting $k s^{2}=0$ in (18) gives $u=\frac{1}{2}$. Having obtained this we write $u=\frac{1}{2}+\varepsilon$, where $\varepsilon \rightarrow 0$ and put this in (18) for further bootstrapping. We get

$$
2 \varepsilon+48 k s^{2}\left(\frac{1}{2}+\varepsilon\right)^{2}\left(\frac{1}{2}-\varepsilon\right)^{2}=0 .
$$

Since the linear term in $\varepsilon$ must match $3 k s^{2}$ we conclude that $\varepsilon=-\frac{3}{2} k s^{2}$ and

$$
u=\frac{1}{2}-\frac{3}{2}\left(k s^{2}\right)+o\left(k s^{2}\right) .
$$

Perform a change of variable $\tau=\operatorname{suv}$ in (17) by taking $v$ as the new integration variable. This gives

$$
(-i) e^{-\pi s / 12} s^{2} u^{3 / 2} \int_{L_{1}} \sqrt{v} \sqrt{1-u v} e^{(\pi / 12 s)\left(1 / u v+1 /(1-u v)+48 k s^{2} u v\right)} d v,
$$

where $L_{1}$ is the corresponding line segment passing through the point 1 , the saddle point for the above integral. Note that in (20) the parameter $\pi / 12 s$ is going to infinity and hence we can employ the standard saddle-point method to find the asymptotic approximation. We again concentrate on the main term. Let

$$
g(v):=\frac{1}{u v}+\frac{1}{1-u v}+48 k s^{2} u v .
$$

We have (see, e.g., p. 93 of [9]) as $s \rightarrow 0^{+}$and uniformly for those $k$ with $k s^{2} \rightarrow 0$,

$$
\begin{gathered}
(-i) e^{-\pi s / 12} s^{2} u^{3 / 2} \int_{L_{1}} \sqrt{v} \sqrt{1-u v} e^{(\pi / 12 s)\left(1 / u v+1 /(1-u v)+48 k s^{2} u v\right)} d v \\
\sim(-i) s^{2} u^{3 / 2} \sqrt{1} \sqrt{1-u} e^{(\pi / 12 s) g(1)}\left(\frac{-2 \pi}{(\pi / 12 s) g^{\prime \prime}(1)}\right)^{1 / 2} .
\end{gathered}
$$

The above is obtained by just letting $v=1$ in the formula in the saddle-point method. Note that

$$
g(1)=\frac{1}{u}+\frac{1}{1-u}+48 k s^{2} u, \quad g^{\prime \prime}(1)=\frac{2}{u}+\frac{2 u^{2}}{(1-u)^{3}} .
$$


Rewriting

$$
g(1)=4+\frac{(2 u-1)^{2}}{u(1-u)}+48 k s^{2} u
$$

and using

$$
\frac{(2 u-1)^{2}}{u(1-u)}=36\left(k s^{2}\right)^{2}+o\left(k^{2} s^{4}\right) \quad \text { and } \quad 48 k s^{2} u=48 k s^{2}\left(\frac{1}{2}-\frac{3}{2} k s^{2}+o\left(k s^{2}\right)\right)
$$

we get

$$
g(1)=4+24 k s^{2}-36 k^{2} s^{4}+o\left(k^{2} s^{4}\right) .
$$

Inserting (23) and the second part of (22) into (21) yields

$$
F_{k}\left(e^{-2 \pi s}\right) \sim s^{5 / 2} \frac{\sqrt{3}}{4} e^{(\pi / 3)(1 / s)} e^{2 \pi k s-3 \pi k^{2} s^{3}}
$$

as $s \rightarrow 0^{+}$in $|\arg s|<\pi / 6$ and it holds uniformly for those $k$ such that $k s^{2} \rightarrow 0$. That $s$ stays in the above-mentioned angular region is required because the integration path $L_{1}$ must remain in the valley of steepest descent, a condition that can be easily guaranteed in the subsequent arguments.

4.2. Asymptotics of the Sum (14). We now come back to (14):

$$
\sum_{j=-\infty}^{\infty}(-1)^{j} p\left(n-k-\frac{3 j^{2}+j}{2}\right) p\left(n+k-\frac{3 j^{2}+j}{2}\right)=\frac{1}{2 \pi i} \oint_{|\xi|=\rho} \frac{F_{k}(\xi)}{f(\xi) \xi^{n-k+1}} d \xi,
$$

where $\rho<1$. Let $\xi=e^{-2 \pi s}, \operatorname{Re}(s)>0$, and express the integral in the variable $s$,

$$
\frac{1}{2 \pi i} \oint_{|\xi|=\rho} \frac{F_{k}(\xi)}{f(\xi) \xi^{n-k+1}} d \xi=(-i) \int_{L_{2}} \frac{F_{k}\left(e^{-2 \pi s}\right) e^{2 \pi s n} e^{-2 \pi s k}}{f\left(e^{-2 \pi s}\right)} d s,
$$

where the only constraint on the contour $L_{2}$ is that $\operatorname{Re}\left(L_{2}\right)>0$ and $\operatorname{Im}\left(L_{2}\right)$ is between $\pm \frac{1}{2}$. We choose $\operatorname{Re}\left(L_{2}\right)=1 / 2 \sqrt{2} \sqrt{n}$ for the need of the saddle-point method below. Again for large $n$ the major contribution to the integral on the right comes from an immediate neighborhood of $s=0$. We plug in the expression in (24) for $F_{k}\left(e^{-2 \pi s}\right)$, replace $f\left(e^{-2 \pi s}\right)$ by the expression from its functional equation (16), and infer that as $n \rightarrow \infty$,

$$
\begin{aligned}
\int_{L_{2}} \frac{F_{k}\left(e^{-2 \pi s}\right) e^{2 \pi s n} e^{-2 \pi s k}}{f\left(e^{-2 \pi s}\right)} d s & \sim \int_{L_{2}} \frac{\left(s^{5 / 2}(\sqrt{3} / 4) e^{(\pi / 3)(1 / s)} e^{2 \pi k s-3 \pi k^{2} s^{3}}\right) e^{2 \pi s n} e^{-2 \pi s k}}{\sqrt{s} e^{(\pi / 12)((1 / s)-s)}} d s \\
& \sim \frac{\sqrt{3}}{4} \int_{L_{2}} s^{2} e^{(\pi / 4)(1 / s)+2 \pi s n} e^{-3 \pi k^{2} s^{3}} d s,
\end{aligned}
$$

where the factor $e^{\pi s / 12}$ is unharmfully dropped from the integrand. We now make a change of variable $s=t / 2 \sqrt{2} \sqrt{n}$ to obtain

$$
\frac{\sqrt{3}}{4} \frac{1}{16 \sqrt{2} n^{3 / 2}} \int_{L_{3}} t^{2} e^{(\pi / \sqrt{2}) \sqrt{n}(1 / t+t)} e^{-(3 \pi / 16 \sqrt{2})\left(t^{3} k^{2} / n^{3 / 2}\right)} d t
$$


where the contour $L_{3}$ passes the saddle point $t=1$ because $\operatorname{Re}\left(L_{2}\right)=1 / 2 \sqrt{2} \sqrt{n}$ by the previous choice. Since the above integral is in the standard form of the saddle-point method, by inserting $t=1$ in the saddle-point method formula, we conclude that as $n \rightarrow \infty$ it is asymptotic to

$$
\begin{array}{r}
\frac{\sqrt{3}}{4} \frac{1}{16 \sqrt{2} n^{3 / 2}} 1^{2} e^{\pi \sqrt{2 n}} e^{-(3 \pi / 16 \sqrt{2})\left(k^{2} / n^{3 / 2}\right)} \sqrt{\frac{-2 \pi}{(\pi \sqrt{n} / \sqrt{2}) 2}} \\
=i 3^{1 / 2} 2^{-25 / 4} n^{-7 / 4} e^{\pi \sqrt{2 n}} e^{-(3 \pi / 16 \sqrt{2})\left(k^{2} / n^{3 / 2}\right)} .
\end{array}
$$

Putting all of this together we have as $n \rightarrow \infty$,

$$
\begin{gathered}
\sum_{j=-\infty}^{\infty}(-1)^{j} p\left(n-k-\frac{3 j^{2}+j}{2}\right) p\left(n+k-\frac{3 j^{2}+j}{2}\right) \\
\sim 3^{1 / 2} 2^{-25 / 4} n^{-7 / 4} e^{\pi \sqrt{2 n}} e^{-(3 \pi / 16 \sqrt{2})\left(k^{2} / n^{3 / 2}\right)} .
\end{gathered}
$$

The asymptotics holds uniformly for $k=o(n)$.

5. Conclusion. In this paper we study the asymptotic properties of the weight of overlined parts in overpartitions. These results provide information about the asymptotic number of pairs of partitions which do not share a part and pairs of partitions where the first one is into distinct parts. This study could be generalized in the context of prefabs [5], [15]. Indeed, in a prefab, objects are represented as a sequence of prime objects. Then we can define overprefabs where the last occurrence of a prime object can be overlined. Each object in an overprefab can be decomposed as a pair of objects that do not share a prime object or as a pair of objects where the first one is into distinct prime objects.

For example, it follows from results obtained here that $\bar{p}(2 n, n)$, the number of pairs of partitions of $n$ that do not share a part is asymptotically

$$
\bar{p}(2 n, n) \sim \sqrt{\frac{2}{3}} d(\lfloor 2 n / 3\rfloor) p(\lceil 4 n / 3\rceil) .
$$

We think that such results hold for other prefabs.

Finally, classical results relating coprime and square-free objects are known for integers and monic polynomials over $G F(q)$. We present an integrated approach to these ideas in [1].

Acknowledgment. The authors thank J. P. Allouche for fruitful discussions and ideas.

\section{References}

[1] J.P. Allouche, S. Corteel, W.M.Y. Goh, and P. Hitczenko, Relations between square-free and coprimes, in preparation.

[2] G.E. Andrews, The Theory of Partitions, Cambridge University Press, Cambridge (1984). 
[3] R. Ayoub, An Introduction to the Analytic Theory of Numbers, Mathematical Surveys and Monographs, no. 10, American Mathematical Society, Providence, RI (1963).

[4] L. Bégin, J.F. Fortin, P. Jacob, and P. Mathieu, Fermionic characters for graded parafermions, Nuclear Phys. B, 659 (2003), 365-386.

[5] E.A. Bender and J.R. Goldman, Enumerative uses of generating functions, Indiana Univ. Math. J., 20 (1971), 753-765.

[6] C. Bessenrodt and I. Pak, Partition congruences by involutions, European J. Combin., 25 (2004), 11391149.

[7] S. Casella and R. Berger, Statistical Inference, Wadsforth \& Brooks/Cole, Monterey, CA (1990).

[8] N.R. Chaganty and J. Sethuraman, Strong large deviation and local limit theorems, Ann. Probab., 21 (1993), 1671-1690.

[9] E.T. Copson, Asymptotic Expansions, Cambridge University Press, Cambridge (1967).

[10] S. Corteel, Particle seas and basic hypergeometric series, Adv. in Appl. Math., 31(1) (2003), 199-214.

[11] S. Corteel and P. Hitczenko, Multiplicity and number of parts in overpartitions, Ann. Combin., 8 (2004), 287-301.

[12] S. Corteel and J. Lovejoy, Frobenius partitions and the combinatorics of Ramanujan's ${ }_{1} \psi_{1}$ summation, J. Combin. Theory Ser. A, 97 (2002), 179-183.

[13] S. Corteel and J. Lovejoy, Overpartitions, Trans. Amer. Math. Soc., 356 (2004), 1623-1635.

[14] S. Corteel, J. Lovejoy, and A.J. Yee, Overpartitions and generating functions for generalized Frobenius partitions, in Mathematics and Computer Science III, Trends in Mathematics, Birkhäuser, Boston, MA (2004), pp. 15-24.

[15] S. Corteel, C.D. Savage, H.S. Wilf and D. Zeilberger, A pentagonal number sieve, J. Combin. Theory Ser. A, 82(2) (1998), 186-192.

[16] G.P. Egorychev, Integral Representation and the Computation of Combinatorial Sums, American Mathematical Society, Providence, RI (1984).

[17] W. Feller, An Introduction to Probability and Its Applications, Vol. I, 3rd edn., Wiley, New York (1968).

[18] J.F. Fortin, P. Jacob, and P. Mathieu, Jagged partitions, Preprint; arXiv:math.CO/0310079.

[19] P. Flajolet and R. Sedgewick, An Introduction to the Analysis of Algorithms, Addison-Wesley, Reading, MA (1998)

[20] P. Flajolet and R. Sedgewick, Analytic Combinatorics, Zeroth edn., 3rd printing (2005); http://algo.inria.fr/flajolet/Publications/AnaCombilto9.pdf

[21] G.H. Hardy and E.M. Wright, An Introduction to the Theory of Numbers, 4th edn., Oxford University Press, Oxford (1960).

[22] J. Lovejoy, Gordon's theorem for overpartitions, J. Combin. Theory Ser. A, 103 (2003), 393-401.

[23] J. Lovejoy, Overpartitions and real quadratic fields, J. Number Theory, 106 (2004), 178-186.

[24] J. Lovejoy, Overpartition theorems of the Rogers-Ramanujan type, J. London Math. Soc. (2), 69 (2004), $562-574$.

[25] A.N. Shiryaev, Probability, 2nd edn., Springer, New York (1996).

[26] W. Szpankowski, Average Case Analysis of Algorithms on Sequences, Wiley, New York (2001).

[27] A.J. Yee, Combinatorial proofs of Ramanujan's ${ }_{1} \psi_{1}$ summation and the q-Gauss summation, J. Combin. Theory Ser. A, 105 (2004), 63-77. 